\title{
Effects of Floods on the Incidence of Acute Hemorrhagic Conjunctivitis in Mengshan, China, from 2005 to 2012
}

\author{
Xuena Liu, ${ }^{1}$ Shuo Qiu, ${ }^{2}$ Zhidong Liu, ${ }^{3}$ Dongzhen Chen, ${ }^{1}$ Hui Liu, ${ }^{2}$ and Guoyong Ding ${ }^{1 *}$ \\ ${ }^{1}$ School of Public Health, Shandong First Medical University \& Shandong Academy of Medical Sciences, Taian, P.R. China; ${ }^{2}$ Shandong Yingcai \\ University, Jinan, P.R. China; ${ }^{3}$ School of Public Health, Shandong University, Jinan, P.R. China
}

\begin{abstract}
This study aimed to examine the association between floods and the morbidity of acute hemorrhagic conjunctivitis (AHC) in Mengshan, China. Relying on the longitudinal data, a generalized additive model (GAM) was applied to quantify the relationship between the morbidity of AHC and floods from 2005 to 2012, controlling for other meteorological variables. Years lived with disability (YLDs) and attributable YLDs were used as the measure of the burden of $\mathrm{AHC}$ because of the floods. Multivariable analysis showed that floods were significantly associated with an increased risk of the morbidity of $\mathrm{AHC}$ (rate ratio $[\mathrm{RR}]=2.136,95 \% \mathrm{Cl}: 2.109-2.163$ ). The total YLDs per 1,000 in Mengshan was 0.2001 , although the value in females was higher than that in males ( 0.2351 versus 0.1686$)$. The YLD per 1,000 of AHC in Mengshan was highest between the ages of 5 and 14 years $(0.6530)$, followed by the age of $0-4$ years $(0.3325)$. The attributable YLD per 1,000 of AHC due to the floods in Mengshan was 0.0434 (95\% Cl: 0.0425-0.0442). Our study confirms that floods have significantly increased the risks of $\mathrm{AHC}$ in the selected study area. Females and youngsters may be the vulnerable populations to develop the flood-related disease.
\end{abstract}

\section{INTRODUCTION}

As one of the most common and most severe forms of natural disasters, the occurrence of flood events accounts for up to one half of all natural disasters in the world. ${ }^{1-3}$ Flood events are estimated to increase in frequency and intensity because of rising sea levels and more frequent extreme precipitation events. ${ }^{4,5}$ Many regions of China were susceptible to various natural hazards under the comprehensive action of many factors. ${ }^{6}$ Located in the tropical and subtropical monsoon region, Guangxi frequently suffers from floods. ${ }^{7}$ According to statistics, from 2001 to 2012, a total of $144,968,500$ people were affected by floods in Guangxi, resulting in 795 deaths and 88.224 billion yuan (US $\$ 12.537$ billion) of economic losses.

Acute hemorrhagic conjunctivitis (AHC) is an acute viral eye disease in recent years, which frequently occurs in the global context. The pathogen of $\mathrm{AHC}$ is enterovirus type 70 (EV70) or a coxsackievirus A24 variant $(C A 24 v){ }^{8}$ Symptoms of conjunctivitis include the sudden onset of ocular pain, swelling of the eyelids, red eyes with conjunctival hemorrhaging, and eye discharge. Although $\mathrm{AHC}$ has a good prognosis, it is prone to outbreaks because of its high incidence, high infectivity, and lack of effective vaccine for prevention. Outbreaks are often associated with close contact in the community setting. It generally persists for 3-7 days before resolving spontaneously. ${ }^{9}$ In summary, AHC is a common acute infectious disease that seriously endangers human health and is classified as class $\mathrm{C}$ infection in China. During the epidemic period of AHC, it may lead to the suspension of business, production, or classes. It will not only cause the loss of a large number of labor resources but also seriously affect the political order and economic order of the society. ${ }^{10}$

Influences of floods are widespread and complicated. Many studies suggest that because of floods after heavy rainfall, polluted water is related with waterborne diseases and epidemics, including AHC. ${ }^{11,12}$ The average annual incidence of

\footnotetext{
*Address correspondence to Guoyong Ding, School of Public Health, Shandong First Medical University \& Shandong Academy of Medical Sciences, No. 619, Changcheng Rd., Taian 271016, P.R. China. E-mail: dgy-153@163.com
}

AHC in Guangxi from 2004 to 2008 was 6.12/100,000, accounting for $1.97-14.2 \%$ of the total number of class $C$ infectious diseases in Guangxi. ${ }^{13}$ Located in the east of Guangxi, the incidence of $\mathrm{AHC}$ in Mengshan is higher than that in the other parts of Guangxi. Statistics shows that from 2005 to 2012, there were 1,476 legally reported cases in Mengshan, with an average annual incidence of $74.549 / 100,000 .{ }^{14}$

There are few studies to evaluate the relationship between floods and AHC at home and abroad, and no such studies have been found in Mengshan of Guangxi, which means that the impact of floods on the morbidity of AHC in Mengshan is still unknown. In view of the background summarized previously, this study aimed to quantify the association between the morbidity of AHC and floods from 2005 to 2012 in Mengshan. Results will contribute to have a better understanding of the health effects of floods and assist in developing local strategies to prevent and reduce the burden of $\mathrm{AHC}$ associated with floods.

\section{MATERIALS AND METHODS}

Research area and period. Mengshan is located in the east of Dayao Mountain in the east of Guangxi (Figure 1). It has a longitude of $110^{\circ} 19^{\prime} \mathrm{E}$ and $110^{\circ} 45^{\prime} \mathrm{E}$ and latitude of $23^{\circ} 52^{\prime} \mathrm{N}$ and $24^{\circ} 23^{\prime} \mathrm{N}$, covering an area of $1,280 \mathrm{~km}^{2}$ and hosting a population of 0.19 million. The country has a subtropical monsoonal climate with an annual mean temperature of $24.5^{\circ} \mathrm{C}$. The average annual rainfall is $1,738 \mathrm{~mm}$, the rainy season usually begins in the mid-April and finally in the late August. From 2005 to 2012, severe and constant precipitation caused frequent floods in Mengshan, resulting in great economic losses and a high number of victims. This location, thus, provides an apt setting to explore the association between flooding and AHC. Considering the seasonal distribution of floods and AHC, the flood seasons (periods between April and September) from 2005 to 2012 were chosen as our study periods.

Data collection and management. Disease surveillance data. Ten-day number of AHC cases from 2005 to 2012 (April to September) were obtained from the China Information System for Disease Control and Prevention. Only the cases confirmed clinically and by laboratory tests, including 


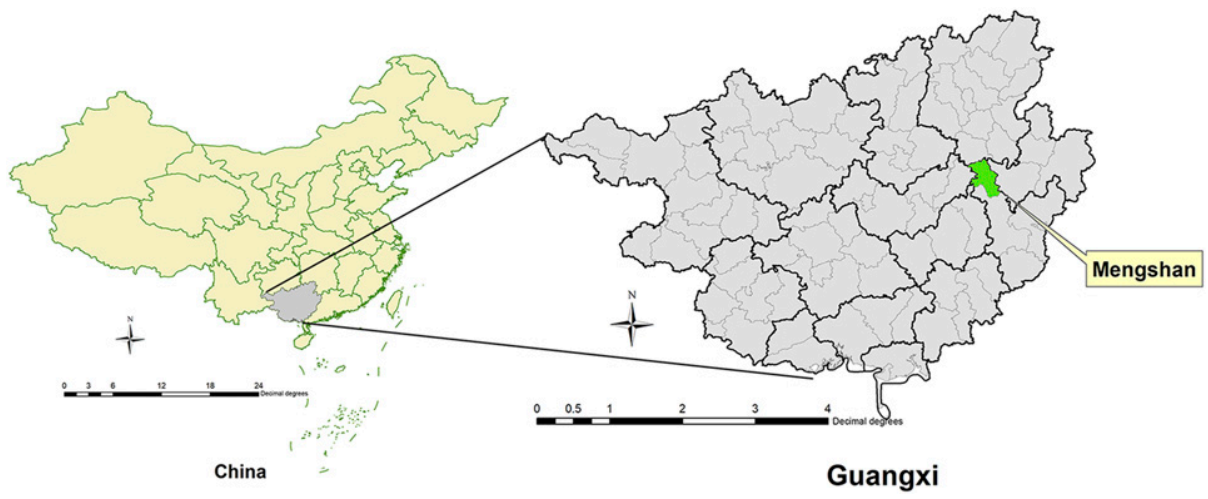

FlGURE 1. Location of Mengshan in Guangxi, China. This figure appears in color at www.ajtmh.org.

microscopic examination and biochemical identification, were included in our study. Information of cases mainly included age, gender, name of disease, type of disease, and date of onset.

Floods data. Flood data were supplied by the yearbooks of meteorological disasters in China, which recorded the number of deaths, damaged areas, occurrence time, and economic loss due to floods. ${ }^{15}$ Based on the meteorological disaster yearbooks, a flood is defined as a natural hazard caused by the overflow of rivers because of sudden constant heavy rainfalls, leading to submerging of villages, lands, fatalities, and economic losses. All flood events which were reported by the yearbooks of meteorological disasters from 2005 to 2012 in the study area were included in the study. During the study period, there were 36 floods recorded in the yearbooks in Menshan, and the flooding lasted 111 days in total.

Meteorological and demographic data. Ten-day meteorological data during the study period were obtained from the China Meteorological Data Service Center (http://data.cma.cn/). The meteorological variables included rainfall, average temperature, minimum temperature, maximum temperature, humidity, wind velocity, air pressure, and sunshine duration. A complete demographic data set was obtained from the Center for Public Health Science Data in China (http://www.phsciencedata.cn/Share/).

Statistical analysis. In our study, we used ten-day data to examine the effects of floods on $\mathrm{AHC}$ based on time series data from 2005 to 2012. During the study period, the ten-day with flooding events was called the flood period, whereas the other ten-day without flooding events or other meteorological disasters was called the non-flood period in this study. The explanation for the "ten-day" time window is shown in Supplemental Figure S1 in the Supplemental Materials.

We first performed a descriptive analysis, and the rate ratio (RR) of morbidity of AHC during the flood and non-flood periods was applied to evaluate the relationship between floods and the morbidity of $\mathrm{AHC}$ with different lagged values. Spearman's rank correlation was adopted to examine the association between climatic variables and the morbidity of $\mathrm{AHC}$. The lagged value with the maximum correlation coefficient for each climate variable was selected for inclusion in the subsequent regression models. Based on the latent period of $\mathrm{AHC}$ and the living habits of the pathogen, a time lag between 0 and 2 ten-days was taken into account.

Second, a generalized additive model (GAM) was established to quantify the association between floods and the tenday incidence of $\mathrm{AHC}$, controlled for meteorological variables. The method of GAM can be used to model seasonality and long-term trend nonparametricallity, and it allows nonparametric and parametric functions to be studied jointly. ${ }^{16}$ It has been widely applied in the studies of the association between meteorological variables and health outcomes. $^{17}$ Excessive dispersion may occur in Poisson regression because of the clustering nature of the $\mathrm{AHC}$ disease. A quasi-Poisson regression was used to deal with the over dispersion of Poisson distribution. In the study, new cases of AHC were not detected in several flood and non-flood periods, and this would result in a large deviation between the estimated results of model parameters and the actual value. Therefore, a very small value was added to the morbidity of $\mathrm{AHC}$. Considering the high correlation between the minimum temperature, maximum temperature, and average temperature, the model containing the average temperature was chosen as the main model. Models with minimum or maximum temperatures were selected as alternative models, which are presented in the supplementary materials. In addition, there is a high correlation between rainfall and flooding also. Therefore, two GAMs were established to examine the association between floods and the morbidity of $\mathrm{AHC}$, that is, the model with rainfall adjusted and the model with rainfall unadjusted. Because the results did not change significantly after adjusted for rainfall, the model with rainfall unadjusted was selected as the final main model and the model with rainfall adjusted has been moved to the Supplemental Materials. The final main model is described as follows:

$$
\ln \left[E\left(Y_{t}\right)\right]=\alpha+\beta \text { flood }+s(\text { tav })+s(\text { hum })+s(\text { sun })+s(\text { time }) \text {, }
$$

where $Y_{t}$ denotes the ten-day number of $\mathrm{AHC}$ cases at time $t$. Flood is a categorical variable including the ten-day of no meteorological disasters, the ten-day of floods, and the tenday of other meteorological disasters, which were represented by 0,1 , and 2 , respectively. $s$ (tav), $s$ (hum), $s$ (rain), and $s$ (sun) are smooth functions of average temperature, humidity, rainfall, and sunshine duration, respectively, which were designed to control the effect of confounding meteorological factors. To avoid the effect of long-term tendency and seasonality, the smooth spline of specific ten-day was projected as $s$ (time). In our study, "s ( $x$ )"was used to fit the GAM by gam function. The appropriate degrees of freedom (DFs) of smooth fitting were found by the method of minimum generalized cross-validation (GCV) score, that is, to determine the DFs based on the minimum GCV score.

As effect estimates vary with different choices of model selections and parameters specification, we conducted the 


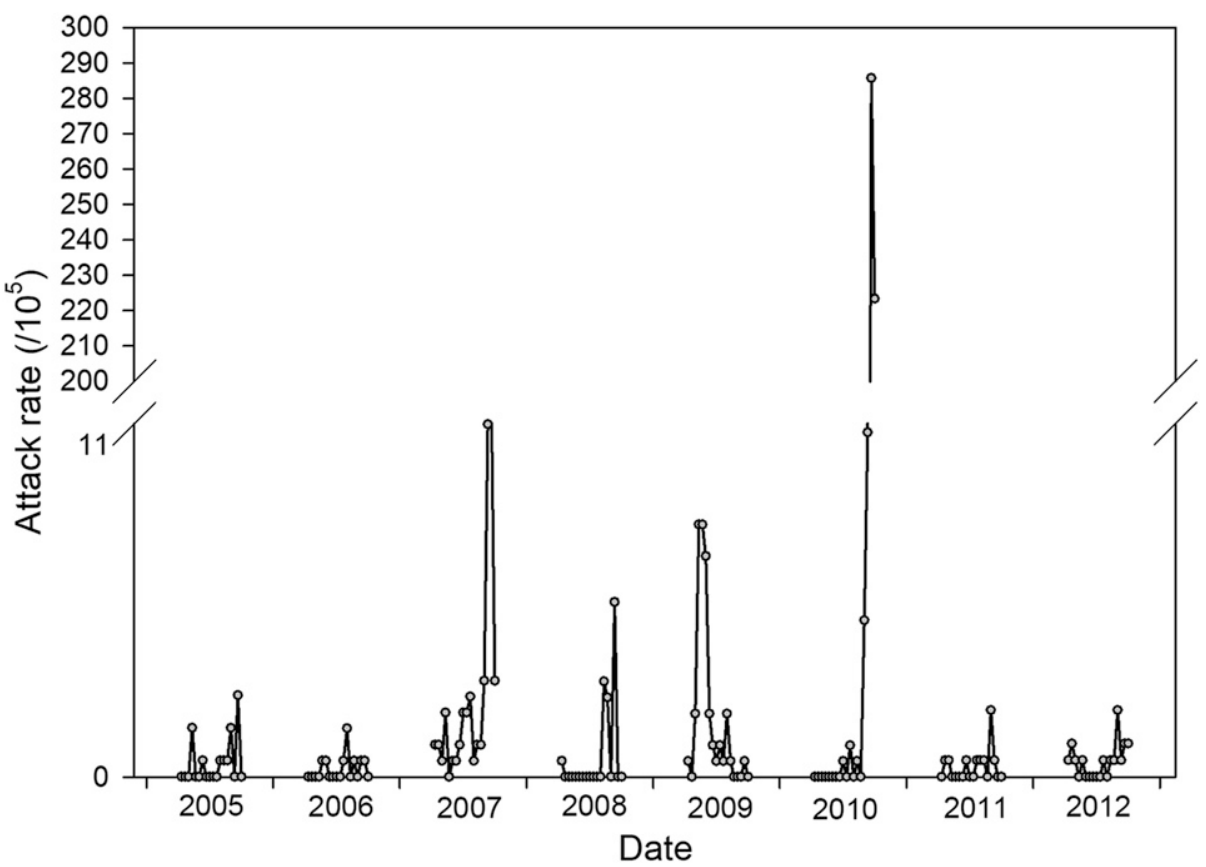

FIGURE 2. Ten-day attack rate of acute hemorrhagic conjunctivitis during the study period from 2005 to 2012 in Mengshan, China.

following sensitivity analyses based on estimated DFs of the GAM: 1) varying the DF (6-11) for time to adjust for long-term trend and seasonality, 2) changing the DF (2-7) for average temperature, 3) changing the DF (6-11) for humidity, and 4) changing the DF (1-6) for rainfall and sunshine duration.

Third, years lived with disability (YLDs) and attributable YLDs were calculated to estimate the burden of $\mathrm{AHC}$ due to the floods. The method of estimating YLDs as recommended by the WHO was used to calculate the burden of $\mathrm{AHC}$ during exposure effect period of flooding. ${ }^{18}$ Calculations of YLDs and YLD per 1,000 were made using DisMod II (WHO, 2001) and Microsoft Office Excel 2003 (Microsoft Corp, Redmond, WA).

Last, the population attributable risk percent (PAR\%) and attributable YLDs were estimated for the percentage of the burden of disease due to $\mathrm{AHC}$ that was attributed to floods. The formula for PAR\% is as follows: ${ }^{19}$

$$
\mathrm{PAR} \%=\frac{P_{e}(\mathrm{RR}-1)}{P_{e}(\mathrm{RR}-1)+1} \times 100 \%
$$

where $P_{e}=$ proportion of exposed population and $\mathrm{RR}=$ relative risk.

The YLDs for the population were multiplied by PAR\% to calculate the fraction of $\mathrm{AHC}$ attributable to floods for the study population, as shown in the following equation. ${ }^{20}$

Attributable YLDs $=\mathrm{PAR} \% \times$ YLDs

\section{RESULTS}

Descriptive analysis for the disease. In total, 1,070 cases of $\mathrm{AHC}$ were diagnosed in Mengshan from 2005 to 2012 with a mean annual incidence of 6.08/10,000. Figure 2 shows the ten-day AHC incidence distribution over the study period. As can be observed from the figure, there were a total of four outbreaks of $\mathrm{AHC}$ during the study period, and the monthly incidence peaked in September 2010 (196.386/100,000). Furthermore, a distinct seasonal trend was observed with more $\mathrm{AHC}$ cases that occurred in summer and autumn.

Lag effect analysis. Figure 3 shows the RR of morbidity of $\mathrm{AHC}$ during the flood and non-flood periods with different lagged values. The ten-day incidence of $\mathrm{AHC}$ correlated positively with the floods with a relevant lag time of 0 ten-day. Table 1 lists the results of Spearman's correlation analysis. Rainfall and humidity correlated negatively with the morbidity of $\mathrm{AHC}$ with a lag time of 1 ten-day. The average temperature, minimum temperature, maximum temperature, and sunshine duration correlated positively with the morbidity of $\mathrm{AHC}$ with a lag time of 2 ten-day. No correlations were found between the ten-day incidence of $\mathrm{AHC}$ and the wind velocity or air pressure. The lagged effects of all meteorological factors were incorporated into further regression analysis.

Regression analysis. The parameters of the GAM are presented in Table 2. After controlling for the other meteorological variables, ten-day incidences of $\mathrm{AHC}$ were in a positive correlation with the floods ( $\mathrm{RR}=2.136,95 \% \mathrm{Cl}: 2.109-2.163)$. The observed morbidity of AHC fitted well with the figures

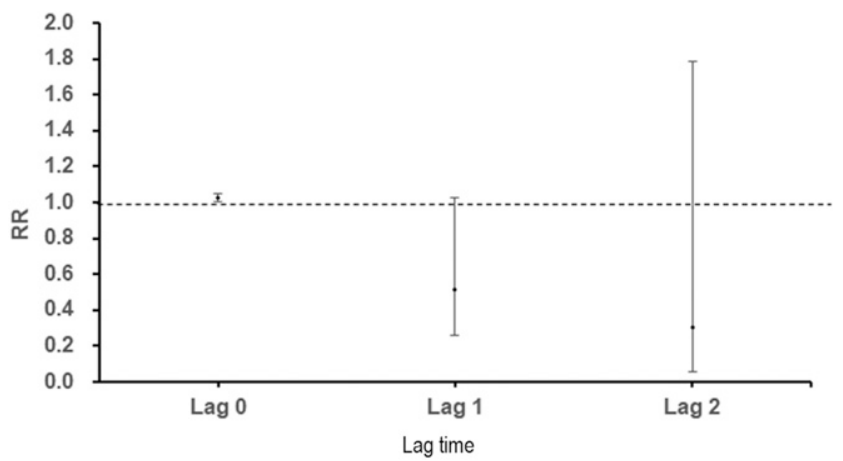

FIGURE 3. Rate ratio of morbidity of acute hemorrhagic conjunctivitis during the flood and non-flood periods with different lagged values. 
TABLE 1

Correlations between the morbidity of acute hemorrhagic conjunctivitis and explanatory variables among ten-day data in Mengshan from 2005 to 2012

\begin{tabular}{|c|c|c|c|c|c|c|c|}
\hline Variable & Lag & $r_{s}$ & $P$ & Variable & Lag & $r_{s}$ & $P$ \\
\hline \multirow[t]{3}{*}{ Rainfall } & 0 & 0.090 & 0.285 & Wind velocity & 0 & 0.015 & 0.860 \\
\hline & 1 & 0.217 & 0.009 & & 1 & 0.111 & 0.187 \\
\hline & 2 & 0.126 & 0.133 & & 2 & -0.064 & 0.445 \\
\hline \multirow[t]{3}{*}{ Average temperature } & 0 & 0.099 & 0.240 & Air pressure & 0 & -0.017 & 0.837 \\
\hline & 1 & 0.210 & 0.012 & & 1 & -0.069 & 0.409 \\
\hline & 2 & 0.235 & 0.005 & & 2 & -0.109 & 0.195 \\
\hline \multirow[t]{3}{*}{ Minimum temperature } & 0 & 0.035 & 0.680 & Sunshine duration & 0 & 0.172 & 0.039 \\
\hline & 1 & 0.148 & 0.077 & & 1 & 0.218 & 0.009 \\
\hline & 2 & 0.175 & 0.036 & & 2 & 0.300 & 0.000 \\
\hline \multirow[t]{3}{*}{ Maximum temperature } & 0 & 0.120 & 0.150 & Humidity & 0 & -0.219 & 0.008 \\
\hline & 1 & 0.222 & 0.007 & & 1 & -0.246 & 0.003 \\
\hline & 2 & 0.247 & 0.003 & & 2 & -0.208 & 0.012 \\
\hline
\end{tabular}

predicted by the developed regression model, with a goodness of fit of $90.2 \%$ (adjusted $R^{2}$ was 0.902 ) (Figure 4).

In the models incorporating maximum temperature (models S2 and S3), the RR value in the rainfall-adjusted model was larger than that in the rainfall-unadjusted model $(R R=2.960$ versus $R R=2.509$ ), although the same cases were found between the two models incorporating minimum temperature $(R R=1.906$ versus $R R=1.873)$. In the rainfall-adjusted models (models S1, S2, and S4), the RR value in the model incorporating maximum temperature was larger than that in the model incorporating minimum temperature and in the model incorporating average temperature $(R R=2.960$ versus $R R=$ 1.906 and $R R=2.124$ ) (the difference of $R R$ between Model S4 and Model S1 was small). And in the rainfall-unadjusted models (the final main Model, Model S3, and Model S5), the same cases were observed $(R R=2.509$ versus $R R=1.873$ and $R R=2.136)$.

In addition, the results of sensitivity analyses showed that the effects estimates did not change substantially when changing the DF for the research variables (Supplemental Figures S2 and S3 in the supplemental Materials).

Analysis for YLDs and attributable YLDs of AHC. Years lived with disability per 1,000 of $\mathrm{AHC}$ among age-gender groups in the period of floods are presented in Table 3. The total YLD per 1,000 in Mengshan was 0.2001 , although the value of female was higher than that of male $(0.2351$ versus 0.1686$)$. The YLD per 1,000 of $\mathrm{AHC}$ in Mengshan was highest between the ages of 5 and 14 years $(0.6530)$, followed by the age of $0-4$ years $(0.3325)$.

We calculated the proportion of the study population in exposing these floods at $23.5 \%$ according to the average number of people affected by the floods in Mengshan. Figure 5 shows the YLDs per 1,000 and the attributable YLDs per 1,000 in the flood period. The attributable YLD per 1,000 in Mengshan was 0.0434 (95\% Cl: 0.0425-0.0442).

\section{DISCUSSION}

Acute hemorrhagic conjunctivitis was first recognized in 1969 with an outbreak in Ghana, Africa. ${ }^{21}$ This disease, which belongs to a new type of acute viral eye disease, has had

TABLE 2

Parameter coefficients from the generalized additive model for the acute hemorrhagic conjunctivitis disease in Mengshan

\begin{tabular}{lcccccc}
\hline Variable & $\beta$ & $\mathrm{SE}$ & $t$ & $P$ & $\mathrm{RR}$ & $95 \% \mathrm{Cl}$ for RR \\
\hline Intercept & 5.207 & 0.007 & 695.14 & $<0.001$ & - & - \\
Floods & 0.759 & 0.006 & 117.38 & $<0.001$ & 2.136 & $2.109-2.163$ \\
\hline $\mathrm{RR}=$ relative risk. & & & & &
\end{tabular}

several pandemics around the world. ${ }^{21-25}$ This study has, for the first time, quantified the association between floods and $\mathrm{AHC}$ in Mengshan, China. The results of all the models confirmed that flood periods would bring more morbidity of $\mathrm{AHC}$ than non-hazard periods in the study areas after adjustment for meteorological factors, seasonality, and long-term trend, which has been reported in both home and abroad. For example, according to the survey conducted by Bich et al. ${ }^{11}$ in $\mathrm{Hanoi}, \mathrm{AHC}$ is a kind of acute intestinal infection disease with a high post-disaster morbidity in hard-hit communities. In 1998, the regional flood in Hubei Province led to the outbreak of $\mathrm{AHC}$ in Jianli County. ${ }^{26}$ A study conducted in Anhui showed that $\mathrm{AHC}$ is one of the major infectious diseases among the patients affected by floods. ${ }^{27} \mathrm{~A}$ study on the epidemic characteristics of disease in Pakistan during the post-flood period found that conjunctivitis is one of the common eye diseases caused by floods. ${ }^{28}$ Similar results were obtained from our study. The findings indicate that floods could be an independent risk factor for $\mathrm{AHC}$ incidences after the adjustment of potential confounders. However, the underlying mechanisms by which floods influence the $\mathrm{AHC}$ are not yet clear. The possible reasons are as follows. First, several studies have shown that floods may have adverse effects on water quality and subsequently lead to enteric infectious diseases. ${ }^{26,29,30}$ Excessive or heavy rainfall events can mobilize pathogens in the environment into rivers, coastal waters, and wells. Such events can, therefore, adversely affect water sources and supply systems, and the transmission of enteric pathogens is likely to be increased. ${ }^{19,30}$ Second, most of the residents after the floods lived in temporary centralized settlements, which were characterized by dense population, crowded living conditions, and humid climate. The poor living conditions and personal hygiene make victims more susceptible to $\mathrm{AHC}$. Third, many disaster areas will strengthen the monitoring of key populations and diseases after floods, and measures will be taken to disinfect the water sources and drinking water in a timely manner. However, a study has shown that such precautions only help cut off the spread of diarrhea or bacterial dysentery and have little effect on other types of dermatitis, conjunctivitis, cold etc. ${ }^{12}$ In addition, some characteristics of Mengshan provide more chances for the spread of AHC. Mengshan is a typical large agricultural county. According to the census data of 2010, the resident population of Mengshan is 193,000 , which included 165,100 agricultural people. Food and drinking water may be contaminated by the unhealthy life style of farmers. To our knowledge, the fragile public health 


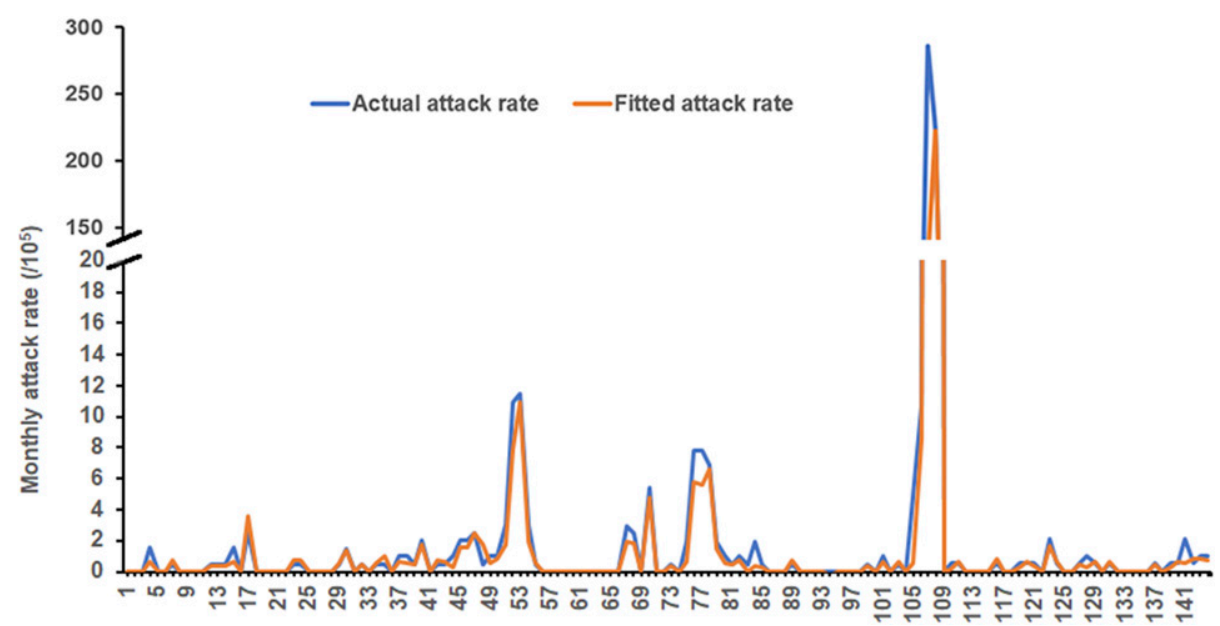

FIGURE 4. Actual attack rate vs. model fitted attack rate and validation by the generalized additive model (adjusted $R^{2}$ was 0.902 ). This figure appears in color at www.ajtmh.org.

system in rural areas and the life styles of local residents may be the most important contributors to the transmission of the pathogens. In addition, one study has shown that high temperatures and rainfall were two climatic factors involved in the rapid spread of pathogens for $\mathrm{AHC} .^{31}$ In our study, we found that the RRs in the models incorporating maximum temperature or rainfall were relatively larger. It means that rainfall and high temperature could increase the risk of developing $\mathrm{AHC}$ by accelerating the spread of pathogens for AHC.

The results of the lag effect suggested that an impact of floods could increase the risk of AHC immediately (lag time was 0 ten-day), considering the incubation period of the disease. Accordingly, it is extremely important for public health practitioners to begin the response just after the flood to prevent and reduce future risks of enterovirus $(E-70)$ infection.

It is important to estimate YLDs in the burden of infectious disease studies, particularly for those infectious diseases that have few deaths and high incidence or prevalence. ${ }^{32}$ Acute hemorrhagic conjunctivitis is one such case. To estimate the burden of $\mathrm{AHC}$ during the flood periods, we have adopted the comprehensive measurement-YLDs, which have been widely adopted by the national and global burden of disease studies for both chronic and infectious diseases. The results suggested that the total YLD per 1,000 in Mengshan was 0.2001 , and the value of female was higher than that of male. A possible explanation is that most of the young and middle-aged men went out to work in the flooding periods, and the heavy work of flood relief and

TABLE 3

Epidemiological burden of acute hemorrhagic conjunctivitis during exposure effect period for floods

\begin{tabular}{lrrcrr}
\hline & & & \multicolumn{3}{c}{ Years lived with disability per 1,000} \\
\cline { 4 - 6 } Age (years) & Case & Attack rate $\left(1 / 10^{5}\right)$ & Males & Females & Persons \\
\hline $0-4$ & 51 & 355.401 & 0.3957 & 0.2638 & 0.3325 \\
$5-14$ & 106 & 434.676 & 0.6925 & 0.6075 & 0.6530 \\
$15-29$ & 92 & 168.335 & 0.1562 & 0.3466 & 0.2451 \\
$30-44$ & 85 & 166.230 & 0.0496 & 0.1783 & 0.1104 \\
$45-59$ & 43 & 120.115 & 0.0142 & 0.0501 & 0.0311 \\
$60-69$ & 19 & 140.782 & 0.0058 & 0.0190 & 0.0120 \\
$70-79$ & 9 & 103.401 & 0.0025 & 0.0033 & 0.0029 \\
$80+$ & 2 & 78.247 & 0.0009 & 0.0009 & 0.0009 \\
Total & 407 & 198.461 & 0.1686 & 0.2351 & 0.2001 \\
\hline
\end{tabular}

production was undertaken by women, which leads to the increase of women's access to contaminated water. This may account for the higher number of female cases. In the agespecific analysis, we have identified a larger effect of floods on $\mathrm{AHC}$ among youngsters than older people, providing evidence that youngsters may be the vulnerable population to develop flood-related AHC. The possible reasons are as follows. First, the youngsters enjoy playing in the water during the floods, which will increase their chances of being infected with pathogens. Second, as described previously, Mengshan is a large agricultural county. Poor infrastructure in rural schools, crowded classrooms and dormitories, as well as poor personal hygiene habits all these could make the young children susceptible to AHC. The last reason why the youngsters are more likely to be injured during natural disasters may be their underdeveloped or compromised immune systems.

Two limitations of this study must also be acknowledged. On the one hand, some confounding factors such as different immune levels, family income, availability of health services, and their individual behaviors that may affect the morbidity of $\mathrm{AHC}$ had not been included in our study. On the other hand, underreporting bias is inevitable. The reported cases included in this study are those who presented serious symptoms and were diagnosed in hospitals. Cases with mild clinical

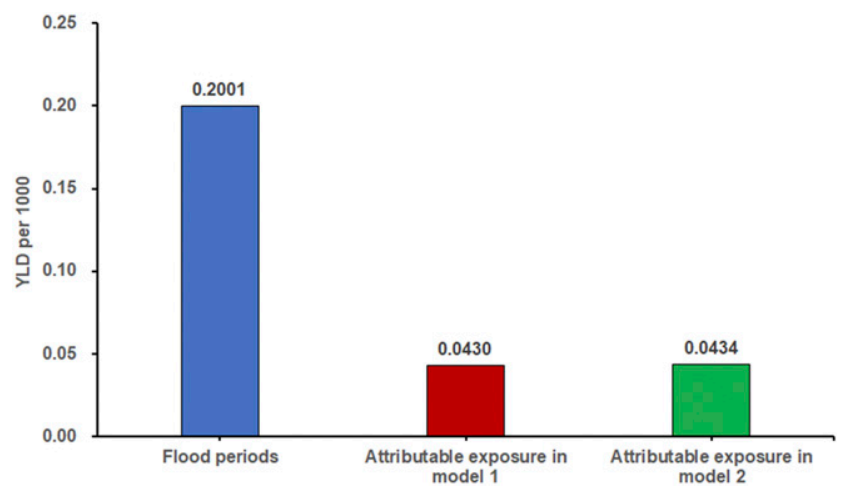

FIGURE 5. Years lived with disability (YLDs) per 1,000 and attributable YLD per 1,000 of acute hemorrhagic conjunctivitis caused by floods in Mengshan during 2005-2012. This figure appears in color at www.ajtmh.org. 
symptoms and treated by themselves usually did not seek health serves, which may lead to an underestimate of the risk of floods. Therefore, the burden of AHC may be much higher than our estimates in this study, if underreporting is corrected.

In conclusion, our study provides strong evidence that flooding is positively associated with the risk of $\mathrm{AHC}$ in the selected study area. Females and youngsters may have more risks to develop the flood-related disease. The findings have significant implications for developing local strategies and measures to prevent and reduce health impact of floods.

Received February 25, 2019. Accepted for publication February 24, 2020.

Published online March 30, 2020.

Note: Supplemental materials and figures appear at www.ajtmh.org.

Acknowledgments: We thank the Chinese Center for Disease Control and Prevention, National Meteorological Information Center of China, and Data Center for Institute of Geographic Sciences and Natural Resources Research of China for sharing the data needed for this study.

Financial support: This study was supported by Ph.D. Scientific Research Staring Foundation of Shandong First Medical University \& Shandong Academy of Medical Sciences (X. L.), the Shandong Province Higher Educational Young and Innovation Technology Supporting Program (2019KJL004), and the Academic Promotion Programme of Shandong First Medical University (2019RC010).

Authors' addresses: Xuena Liu, Dongzhen Chen, and Guoyong Ding, School of Public Health, Shandong First Medical University \& Shandong Academy of Medical Sciences, Taian, P.R. China, E-mails: xuena_liu@ 163.com, 965565807@qq.com, and dgy-153@163.com. Shuo Qiu and Hui Liu, Shandong Yingcai University, Jinan, P.R. China, E-mails: huiliu_dd@ 163.com and 1090695616@qq.com. Zhidong Liu, School of Public Health, Shandong University, Jinan, P.R. China, E-mail: liuzhidong3105@163.com.

\section{REFERENCES}

1. Feng $S$ et al., 2007. Social support and posttraumatic stress disorder among flood victims in Hunan, China. Ann Epidemiol 17: 827-833.

2. Alderman K, Turne LR, Tong S, 2012. Floods and human health: a systematic review. Environ Int 47: 37-47.

3. Wakuma Abaya S, Mandere N, Ewald G, 2009. Floods and health in gambella region, Ethiopia: a qualitative assessment of the strengths and weaknesses of coping mechanisms. Glob Health Action 2: 2019.

4. Ramin B, McMichael A, 2009. Climate change and health in subSaharan Africa: a case-based perspective. Ecohealth 6: 52-57.

5. Cao L, Zhong J, Su B, Zhai J, Gemmer M, 2013. Probability distribution and projected trends of daily precipitation in China. Adv Clim Change Res 4: 153-159.

6. Kosek M, Bern C, Guerrant R, 2003. The global burden of diarrhoeal disease, as estimated from studies published between 1992 and 2000. Bull World Health Organ 81: 197-204.

7. Zhang Y, Bi P, Sun YW, Hiller JE, 2012. Projected years lost due to disabilities (YLDs) for bacillary dysentery related to increased temperature in temperate and subtropical cities of China. $J$ Environ Monit 14: 510-516.

8. Wang X, Zhao J, Zhang J, Zhang S, Si B, Wang Y, 2014. Acute hemorrhagic conjunctivitis outbreak in China. Dis Surveill 29: 92-97.

9. Kurokawa M, Rai SK, Ono K, Gurung R, Ruit S, 2006. Viral investigation of acute hemorrhagic conjunctivitis outbreak (2003) in Nepal using molecular methods. Southeast Asian J Trop Med Public Health 37: 904-910.

10. Zhang X, Liu G, Pan Q, Li L, Chen L, Yang G, 2018. Epidemic characteristics of acute hemorrhagic conjunctivitis in Wenzhou city. Prev Med 30: 816-818.

11. Bich TH, Quang LN, Ha leTT, Hanh TT, Guha-Sapir D, 2011. Impacts of flood on health: epidemiologic evidence from Hanoi, Vietnam. Glob Health Action 4: 6356.
12. Wang M, 2000. The distribution and characteristics of flood related diseases in the Yangtze river basin. Dis Reduct China 10: 38-42.

13. Chang Z, Zhang J, Wang Z, 2009. Analysis on epidemic feature of acute hemorrhagic conjunctivitis from 2004 to 2007 in China. Chin J PHM 25: 268-270.

14. Data center for Public Health, 2006-2013. National Notifiable Infectious Disease Database [in Chinese]. Available: http:// www.phsciencedata.cn/Share/ky_sjml.jsp. Accessed December 16, 2018.

15. China Meteorological Administration, 2006-2013. The Yearbooks of Meteorological Disasters in China. Beijing, China: China Meteorological Press.

16. Schnitzler J, Benzler J, Altmann D, Mucke I, Krause G, 2007. Survey on the population's needs and the public health response during floods in Germany 2002. J Public Health Manag Pract 13: 461-464.

17. Zhang Y, Peng L, Kan H, Xu J, Chen R, Liu Y, Wang W, 2014. Effects of meteorological factors on daily hospital admissions for asthma in adults: a time-series analysis. PLoS One 9: e102475.

18. Mathers C, Vos T, Lopez A, Salomon J, Ezzati M, 2001. National Burden of Disease Studies: A Practical Guide, 2.0 edition. Geneva, Switzerland: Global Program on Evidence for Health Policy. World Health Organization. Available: http://www.who.int/entity/ healthinfo/nationalburdenofdiseasemanual.pdf. Accessed February $11,2019$.

19. Cole $\mathrm{P}$, MacMahon $\mathrm{B}, 1971$. Attributable risk percent in casecontrol studies. Br J Prev Soc Med 25: 242-244.

20. Prüss-Üstün A, Mathers C, Convalán C, Woodward A. 2003. Introduction and Methods: Assessing the Environmental Burden of Disease at National and Local Levels. Geneva, Switzerland: World Health Organization. (WHO Environmental Burden of Disease Series, No.1). Available: https://www.who.int/quantifying_ehimpacts/ publications/en/9241546204.pdf. Accessed February 12, 2019.

21. Chatterjee S, Quarcoopome CO, Apenteng A, 1970. Unusual type of epidemic conjunctivitis in Ghana. Br J Ophthalmol 54: 628-630.

22. Tavares FN, Campos Rde M, Burlandy FM, Fontella R, de Melo MM, da Costa EV, da Silva EE, 2011. Molecular characterization and phylogenetic study of coxsackievirus A24v causing outbreaks of acute hemorrhagic conjunctivitis (AHC) in Brazil. PLoS One 6: e23206.

23. Shukla D, Kumar A, Srivastava S, Dhole TN, 2013. Molecular identification and phylogenetic study of coxsackievirus A24 variant isolated from an outbreak of acute hemorrhagic conjunctivitis in India in 2010. Arch Virol 158: 679-684.

24. Wu B, Qi X, Xu K, Ji H, Zhu Y, Tang F, Zhou M, 2014. Genetic characteristics of the coxsackievirus A24 variant causing outbreaks of acute hemorrhagic conjunctivitis in Jiangsu, China, 2010. PLoS One 9: e86883.

25. Ayoub EA, Shafik CF, Gaynor AM, Mohareb EW, Amin MA, Yassin AS, El-Refaey S, Genedy M, Kandeel A, 2013. A molecular investigative approach to an outbreak of acute hemorrhagic conjunctivitis in Egypt, October 2010. Virol J 10: 96.

26. Zeng G, Chen W, Zhang X, 2005. The influence of 1998 flooding on infectious diseases epidemiology in Hubei province. Pract Prev Med 12: 249-252.

27. Shi Q, 2004. Investigation of diseases distribution of flood victims in a township in 2003. Anhui J Prev Med 10: 159-160.

28. Gao H, Liang L, Zhang K, Liu Q, Chen X, Li X, 2010. Problems and strategies of classification of diseases in international medical rescue during Pakistan flood. Acta Acad Med CPAF 19: 987-991.

29. Liu Z, Li J, Zhang Y, Ding G, Xu X, Gao L, Liu X, Liu Q, Jiang, 2016. Distributed lag effects and vulnerable groups of floods on bacillary dysentery in Huaihua, China. Sci Rep 6: 29456.

30. Cann KF, Thomas DR, Salmon RL, Wyn-Jones AP, Kay D, 2013. Extreme water-related weather events and waterborne disease. Epidemiol Infect 141: 671-686.

31. Zhang L, Zhao N, Huang X, Jin X, Geng X, Chan T, Liu S, 2017. Molecular epidemiology of acute hemorrhagic conjunctivitis caused by coxsackie A type 24 variant in China, 2004-2014. Sci Rep 7: 45202.

32. Zhang $Y, B i P$, Janet $E H, 2012$. Projected burden of disease for Salmonella infection due to increased temperature in Australian temperate and subtropical regions. Environ Int 44: 26-30. 